\section{Acute mesenteric ischemia due to superior mesenteric artery embolism in a patient with permanent atrial fibrillation}

\author{
MIROSLAV ŠIMUNIĆ • DAMIR FABIJANIĆ • NIKOLA PERKOVIĆ • \\ ZORAN BOGDANOVIĆ • MARINA MARAS-ŠIMUNIĆ • \\ TONĆI BATINIĆ • ANTE TONKIĆ
}

\author{
MIROSLAV ŠIMUNIĆ $(\bowtie) \bullet$ \\ NIKOLA PERKOVIĆ • \\ ZORAN BOGDANOVIĆ • \\ ANTE TONKIĆ \\ Department of Gastroenterology \\ and Hepatology \\ Split University Hospital Centre \\ Split, Croatia \\ Split University Hospital Centre \\ Spinčićeva 1, 21000 Split, Croatia \\ Phone: +38521556002 \\ Fax: +38521556031 \\ E-mail: ms@kbsplit.hr \\ DAMIR FABIJANIĆ \\ Department of Cardiology \\ Split University Hospital Centre \\ Split, Croatia \\ MARINA MARAS-ŠIMUNIĆ • \\ TONĆI BATINIĆ \\ Department of Radiology \\ Split University Hospital Centre \\ Split, Croatia
}

Key words: thromboembolism, superior mesenteric artery, diagnosis, mesenteric angiography

\section{CASE REPORT}

A 59-year old man was referred to our hospital because of sudden onset of dull, continuous and moderate to severe abdominal pain. The pain was associated with nausea and passage of dark, loose stools. His medical history was significant for permanent atrial fibrillation (AF) associated with alcoholic cardiomyopathy. Because of low thromboembolic risk the patient had not received anticoagulant therapy. On physical examination an irregular rhythm of approximately $120 \mathrm{bpm}$ and a grade 2/6 systolic murmur at the apex was revealed. His arterial blood pressure was 140/85 mmHg. The abdomen was soft and mildly tender to deep palpation, especially in the periumbilical region. Bowel sounds were not reduced.

ECG revealed AF with incomplete right bundle branch block. Chest radiography demonstrated mild cardiomegaly. The plain radiograph of the abdomen showed two air fluid levels in the right inguinal region. Abdominal ultrasound and upper gastrointestinal endoscopy indicated no abnormalities. Laboratory data revealed a raised white cell count $\left(15.95 \times 10^{9} / \mathrm{L}\right.$, normal value 4.0-10.0 x $\left.10^{9} / \mathrm{L}\right)$, creatine kinase (570 UL, normal value $\leq 130 \mathrm{UL}$ ) and lactate dehydrogenase (336 UL, normal value $\leq 240$
UL). Other laboratory parameters were in the normal range.

Because of clinical suspicion for superior mesenteric artery (SMA) emboli$\mathrm{sm}$, the patient underwent contrastenhanced abdominal CT. It showed jejunal luminal dilatation with thickened bowel wall. The adjacent mesenterium showed increased density of the fat tissue and selective congestion. One SMA branch showed a partial filling defect while one of the left branches had a complete loss of circulation. The finding was interpreted as an embolic occlusion with subsequent changes in the jejunum (figures 1 and 2).

Selective arteriography of the celiac trunk and SMA showed smaller filling defects in the borderline segment 
between the proximal and middle parts of the SMA as well as in the first part of the jejunal branch with signs of adequate luminal recanalization (figure 3 ).

An abdominal surgeon was consulted and conservative treatment was recommended. Treatment with subcutaneous analgesics, oxygen, intravenous broad-spectrum antibiotics and fluid resuscitation with crystalloids (50-70 $\mathrm{mL} / \mathrm{kg}$ daily), according to hemodynamic monitoring, was commenced. A nasogastric tube was inserted to relieve distension.

Transthoracic echocardiography demonstrated slightly increased left atrial $(L A=42 \mathrm{~mm})$ and left ventricular (LVIDd $=58 \mathrm{~mm}$ ) diameters as well as slightly reduced left ventricular systolic function $(\mathrm{LVEF}=46 \%)$. There were no other echocardiographic abnormalities. Transesophageal echocardiography (TEE) (figure 4a) showed a thrombus (10x6 mm) in the left atrial appendage (LAA). A spontaneous echocontrast in the $L A$ and the $L A A$, as well as reduced emptying flow velocity of LAA $(0.22 \mathrm{~m} / \mathrm{s})$ was detected (figure 4b).

There were no definitive signs of bowel necrosis (according to repeated plain abdominal radiographs, laboratory analysis, absence of peritoneal signs, and patient's general condition), therefore, no surgical intervention was necessary. Low-weight molecular heparin was first started simultaneously followed by warfarin within 72 hours. Heparin was discontinued after therapeutic prothrombin time (International Normalized Ratio $=I N R>2.0$ ) had been reached. Clinically improved, the patient was discharged 12 days after admission and permanent anticoagulation, with warfarin, was recommended.

\section{Discussion}

$\mathrm{AMl}$ is a relatively rare medical emergency, with a high mortality rate of $60-90 \%$. It is characterized by the sudden onset of abdominal pain, out of proportion to the physical findings, most frequently in patients with a history of heart disease. Nearly $40-50 \%$ of all AMI cases are caused by embolism of the SMA,

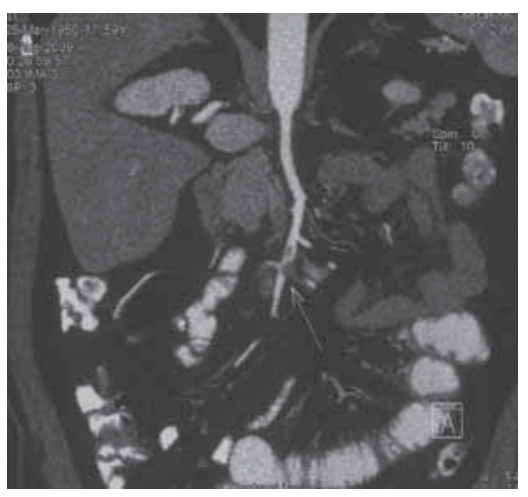

Figure 1. MSCT multiplanar reconstruction (MPR) of the embolisation site.

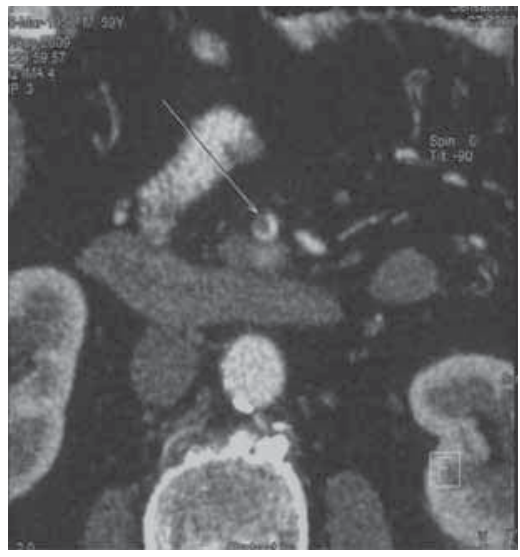

Figure 2. MSCT scan of the embolisation site.

with emboli most commonly originating in the left atrium, particularly in patients with AF. Emboli to the SMA most commonly lodge at points of normal anatomic narrowing usually just distal to the origin of major branches, and may partially or completely occlude the vessel's lumen causing mesenteric ischemia. $(1,2)$ Clinical suspicion, prompt diagnosis and treatment before the onset of bowel infarction may highly reduce mortality.

Thrombi in AF patients almost always originate in the LAA, rather than in the smooth-walled atrium proper. These thrombi are not reliably detected with TEE. $(3,4)$ Thus, in patients with suspected $A M I$ and $A F$, TEE is recommended. (4) Patients with permanent AF form LAA thrombi intermittently. Moreover, TEE reveals the LAA thrombi in only $20 \%$ to $40 \%$ of AF patients with

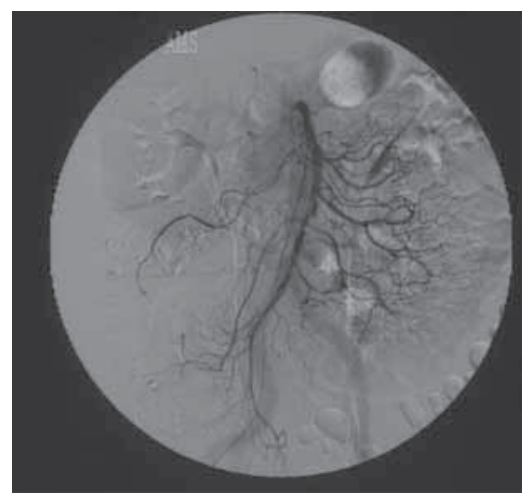

Figure 3. Angiography view of the embolisation site.
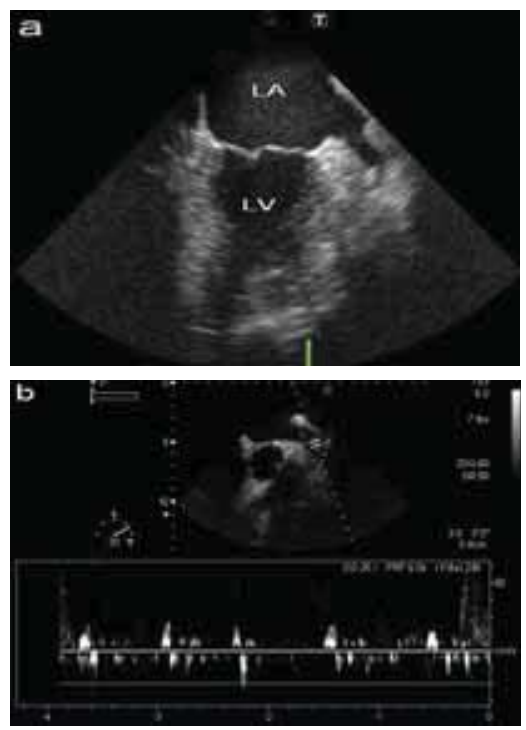

Figure 4. Transesophageal echocardiography demonstrates a thrombus in the left atrial appendage (a), and reduced emptying flow velocity of LAA (b).

recent thromboembolism. (5) The lack of visualization of LAA thrombi on TEE after an embolic event does not exclude the LAA as the embolic source. Because blood stasis is fundamental to the formation of LAA thrombi in AF, the transesophageal echocardiographic readings of blood stasis (i.e. decreased LAA ejection fraction and flow velocity, dense spontaneous echo contrast) are accepted as independent predictors of embolic risk. (3-5)

Once a SMA embolus is suspected a prompt diagnosis of $\mathrm{AMI}$ is needed. Although plain films of the abdomen 
have a low sensitivity and specificity, they are almost always obtained as part of the work-up in such patients. (6) However, early in the course of AMI, an abdominal X-ray is usually normal. Furthermore, standard CT of the abdomen lacks sufficient sensitivity to be used as a diagnostic test for AMI. (7) More recently, multidetector $\mathrm{CT}$ and $\mathrm{MR}$ angiography have replaced plain film and standard CT use, and become the cornerstones of AMI diagnosis. $(7,8)$ MultidetectorCTangiographyrepresents a fast and accurate investigation tool for the diagnosis of AMI. In most cases it can be used as the sole diagnostic procedure. This method provides direct visualization of the mesenteric vasculature, intestines and mesentery. With its high spatial resolution and volumetric three dimensional reconstructions, multidetector CT angiography allows direct non-invasive visualization of the etiology of $\mathrm{AMl}$ and its insults on the intestines. In addition, it can capture associated findings/pathology through the anatomical detail window. Contrast enhanced multidetector CT angiography enables fast and detailed evaluation of the mesenteric circulation and abdominal viscera which provides accurate and rapid AMI diagnosis. Occlusive emboli in the SMA can be readily detected within the lumen and are generally localized beyond the middle colic artery origin, leading to abrupt cessation of contrast material flow within the vessel lumen. Few or no collaterals are seen secondary to the acute nature of the pathology. (7)

Because of its high sensitivity (90-100\%) and specificity (about 100\%), as well as therapeutic potential (possibility of intravascular administration of vasodilatators and thrombolytics) selective catheter angiography is accepted as the gold standard for diagnosis of AMI. $(9,10)$ On angiography, emboli to the SMA are typically manifested as sharp, rounded filling defects in the contrast column with high-grade or subtotal occlusion of distal flow. (9) Vasospasm, distal to the site of embolic occlusion, is frequently present. It is important to assess the development of collateral vessels from the coeliac axis or the inferior mesenteric artery connecting with distal branches when total occlusion of the SMA is seen. (9) Concordantly with CT angiography findings, enhanced collaterals detected with catheter angiography may indicate chronic occlusion of the SMA. $(7,9)$ However, in some cases of AMI rapid establishment of a collateral vessel prevents development of bowel necrosis. Disadvantages of traditional angiography are its limited availability and potential renal toxicity as well as its monetary and time constraints. $(9,10)$

Treatment is dependent upon the type of AMI, but in most situations any patient with peritoneal signs should be operated upon without delay. $(2,6)$ Standard surgical therapy for AMI involves resection of irreparably damaged bowel and reestablishment of mesenteric blood flow through embolectomy. (2) Patients with minor emboli, defined as emboli limited to SMA branches or to the SMA distal to the ileocolic artery, as was revealed in our case, may be managed nonoperatively with volume resuscitation, broad-spectrum antibiotics, vasodilators, and anticoagulants. $(2,9,10)$

As seen in our patient, thromboembolism is one of the major complications of AF. Its prevention continues to be a major challenge in the modern treatment of this type of common arrhythmia. (3-5) However, not all patients with AF will benefit equally from longterm anticoagulation. (5) In AF patients $<75$ years of age and with none of the other thromboembolic risk factors such as hypertension, diabetes mellitus, moderately or severely impaired left ventricular systolic function and/or heart failure, permanent anticoagulant therapy is not recommended because of their low thromboembolic risk. (5) On the other hand, in patients with AF who have had a prior thromboembolic event, long-term anticoagulation with warfarin is mandatory, because of the high risk of future thromboembolism. (5) Therefore, in order to prevent future thromboembolism our patient was discharged with permanent anticoagulation with warfarin (recommended INR is 2.0-3.5).

In conclusion, $\mathrm{AMI}$ is a relatively uncommon cause of abdominal pain, but one with a high mortality rate. Its prompt recognition and aggressive treatment can prevent bowel infarction and improve outcomes. In the proper clinical setting, it is crucial to maintain a high index of clinical suspicion so that a correct diagnosis can be made and treatment initiated expeditiously. 


\section{REFERENCES}

1. Basavanagowdappa H, Babu S, Kumar P, Nanaiah MM, Jeevan HR. Superior mesentric artery embolism. J Assoc Physicians India 2008;56:907-9

2. Barakate MS, Cappe I, Curtin A, Engel KD, Li-Kim-Moy J, Poon MS, et al. Management of acute superior mesenteric artery occlusion. ANZ J Surg 2002;72:25-9.

3. Stoddard MF. Risk of thromboembolism in acute atrial fibrillation or atrial flutter. Echocardiography 2000;17:393-405.

4. Fabijanic D, Giunio L, Vujicic M, Vukovic I, Ermacora R, Knezevic N. Right atrial pacemaker lead thrombosis incidentally detected by transesophageal echocardiography. Coll Antropol 2005;29:161-3.

5. Singer DE, Albers GW, Dalen JE, Fang MC, Go AS, Halperin JL, et al. Antithrombotic therapy in atrial fibrillation: American College of Chest Physicians Evidence-Based Clinical Practice Guidelines (8th Edition). Chest 2008;133:546S-592S.

6. Kozuch PL, Brandt LJ. Review article: diagnosis and management of mesenteric ischaemia with an emphasis on pharmacotherapy. Aliment Pharmacol Ther 2005;21:201-15.

7. Türkbey B, Akpinar E, Cil B, Karçaaltincaba M, Akhan O. Utility of multidetector CT in an emergency setting in acute mesenteric ischemia. Diagn Interv Radiol 2009;15:256-61.

8. Shih MC, Angle JF, Leung DA, Cherry KJ, Harthun NL, Matsumoto AH, et al. CTA and MRA in mesenteric ischemia: part 2, Normal findings and complications after surgical and endovascular treatment. AJR Am J Roentgenol 2007; 188:462-71.

9. Turmezei TD, Cockburn JF. Digital subtraction angiography of the superior mesenteric artery: identifying arterial branches. Clin Anat 2009;22:777-9.

10. Acosta S, Sonesson B, Resch T. Endovascular therapeutic approaches for acute superior mesenteric artery occlusion. Cardiovasc Intervent Radiol 2009;32:896-905. 